\title{
Quantum decay of dark solitons
}

\author{
D.M. Gangardt ${ }^{1}$ and A. Kamenev ${ }^{2}$ \\ ${ }^{1}$ School of Physics and Astronomy, University of Birmingham, Edgbaston, Birmingham, B15 2TT, UK \\ ${ }^{2}$ Fine Theoretical Physics Institute and Department of Physics, \\ University of Minnesota, Minneapolis, MN 55455
}

\begin{abstract}
Unless protected by the exact integrability, solitons are subject to dissipative forces, originating from a thermally fluctuating background. At low enough temperatures $T$ background fluctuations should be considered as being quantized which enables us to calculate finite lifetime of the solitons $\tau \sim T^{-4}$. We also find that the coherent nature of the quantum fluctuations leads to long-range interactions between the solitons mediated by the superradiation. Our results are of relevance to current experiments with ultracold atoms, while the approach may be extended to solitons in other media.
\end{abstract}

PACS numbers: 05.30.Jp,03.75.Kk,03.75.Mn

Soliton dynamics is in a heart of multiple areas of physics and applied mathematics [1]. The recent resurgence of interest in propagation of solitons through a dynamic media 2, 3, was stimulated by a number of experimental observations [4, 5] of dark solitons (DS) in one dimensional (1D) Bose systems. The studies of the soliton dissipative dynamics 2, 3, 6, are probably the most relevant for the current experiments. Indeed the record lifetime of the dark soliton is $\sim 200 \mathrm{~ms}$ [5], calling for understanding of the ultimate quantum limits for soliton persistence.

As follows from the Mermin-Wagner theorem, the mean-field approach is not valid in 1D even at zero temperature. This calls for an essentially quantum description, first achieved by Lieb and Liniger [7] for 1D bosons with the contact two-body interactions, solvable within the Bethe ansatz. As was latter shown by Kulish, Manakov and Faddeev 8 one of the fundamental excitations within the Bethe ansatz classification (so called Lieb II mode [9]) essentially coincides with DS. This fact preserves existence of DS within the Lieb-Liniger model even on the quantum level. The underlying reason for such a stability is the infinite set of conservation laws, characteristic for exactly integrable models 10 . It allows exact canonical transformation to collective coordinates of the soliton and its description as a point-like particle obeying quantum mechanics [11. At $T=0$ the Heisenberg uncertainty of the soliton position and its quantum spreading during the evolution impedes the imaging of dark solitons 12 .

At finite $T$ the soliton loses its quantum coherence and it was realized in Ref. 22 that the major factor limiting DS lifetime is the lack of the exact integrability. In a strongly confined (with transverse frequency $\omega_{\perp}$ ) gas it stems from the virtual transitions to higher states of the transverse quantization leading to the effective three-body contact interactions with the coupling constant $\alpha=-12 \ln (4 / 3) g^{2} /\left(\hbar \omega_{\perp}\right)$ [13. Here $g$ is the strength of delta-like two-body interaction. On the classical level such three-body interactions lead to a cubic
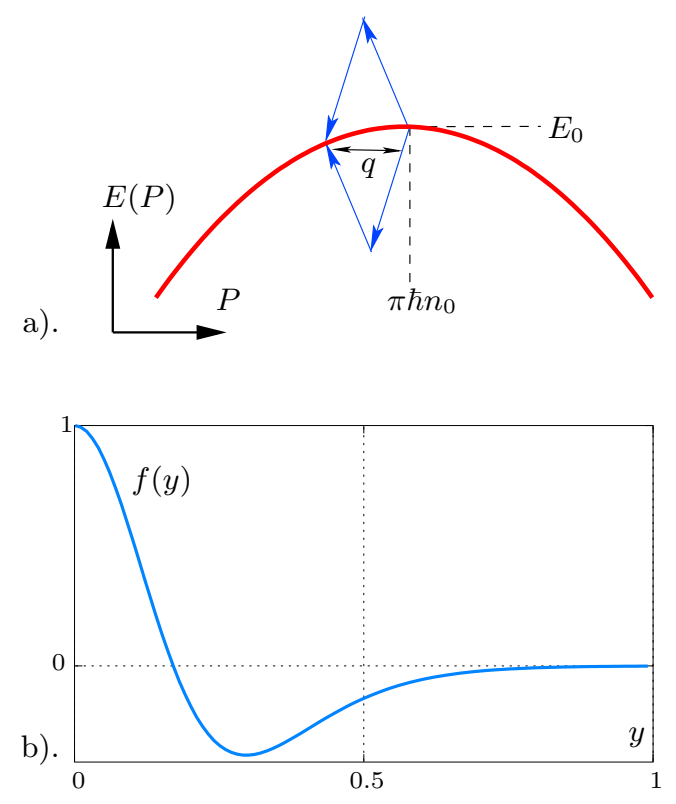

FIG. 1: a) Dispersion relation $E(P)$ of soliton and twophonon processes leading to the dissipation. The arrows represent absorption and emission of long wavelength phonons; their slope is given by sound velocity c. b) Function $f(y)$ defined in Eq. 20.

in density term $\delta L=-\alpha n^{3} / 6$ in GP Lagrangian [2]. The corresponding modified Gross-Pitaevskii (GP) equation still possesses a one parameter family of soliton solutions, however, due to the broken classical integrability, such solitons are unstable against scattering on thermally excited density fluctuations. This classical mechanism of soliton dissipation was shown to result in a finite lifetime $\tau \sim(T / \mu)^{-1}$, for temperatures $T$ higher than chemical potential $\mu$ of $1 \mathrm{D}$ liquid [2]. In this Letter we report a study of the soliton dynamics in the lowtemperature regime $T \ll \mu$. For such low temperatures the phonons are "cold": the density and phase fluctuations of the background need to be treated as quantized objects. Since the soliton velocity $V$ is always smaller 
than the speed of sound $c$, emission of a single phonon is forbidden by the energy and momentum conservation, i.e. by Landau criterion. The leading allowed process is the Raman two-phonon scattering depicted in Fig. 1a, where one thermal phonon is absorbed and another one reemitted. The rate of these processes and their physical consequences in the dynamics of DS constitutes our main results which are summarized in Eqs. (1.4) followed by their derivation.

For small soliton velocities $V \ll c$ the characteristic momentum transferred to phonons $q \sim T / c$ is much less than the typical DS momentum $\pi \hbar n_{0}$ justifying the semilassical treatment and we find the equation of motion

$$
M^{*} \dot{V}=-\kappa V
$$

Here $M^{*}=-4 \hbar n_{0} / c$ is the (negative) effective mass of the soliton, while the right hand side is the viscous friction force due to phonon scattering [14]. Due to the negative effective mass $M^{*}$ the peculiarity of DS dynamics is that the friction force accelerates rather than decelerates the soliton. The accelerated DS looses its energy and eventually thermalizes with the phonons. The quantum nature of the phonons manifests itself in long-range interactions between solitons if one considers a generalization of Eq. 11 for a gas of DS with the set of coordinates $X_{i}$ and velocities $V_{i}$,

$$
M^{*} \dot{V}_{i}=-\kappa(T) \sum_{j} \frac{V_{i}+V_{j}}{2} f\left(\frac{X_{i}-X_{j}}{L_{T}}\right),
$$

where the temperature length is defined as $L_{T}=\hbar c / T$ and the function $f(y)$ is defined in Eq. 20 and shown in Fig. 1p. It is characterized by $f(0)=1$ and $f(y) \approx$ $-30 \pi y e^{-4 \pi y}$ for $y \gg 1$. As a result, only solitons within the distance $X_{i}-X_{j} \lesssim L_{T}$ interact effectively with each other. This is clearly only possible deep in the quantum regime $T \ll \mu$, where $L_{T}$ is much larger than DS size given by the healing length $\xi=\hbar / m c$. Contrary to the usual potential forces, the mutual friction in Eq. (2) is an even function of the relative coordinate, and an odd function of the center of mass velocity. As a result it does not affect the relative motion $M^{*}\left(\dot{V}_{1}-\dot{V}_{2}\right)=\kappa\left(V_{2}-V_{1}\right)$ while the center of mass motion is strongly affected $M^{*}\left(\dot{V}_{1}+\dot{V}_{2}\right)=-\kappa\left(V_{1}+V_{2}\right)\left[1+f\left(\left(X_{1}-X_{2}\right) / L_{T}\right)\right]$. In case $X_{1}-X_{2} \lesssim 0.2 L_{T}$ the two DS accelerate almost twice as fast as a single one. This is a consequence of the superradiation, when phonons emitted by one DS stimulate coherent emission by the other. The same mechanism slows down the acceleration of two DS center of mass for $X_{1}-X_{2} \gtrsim 0.2 L_{T}$, Fig. 1 b.

We found for the viscosity coefficient

$$
\kappa(T)=\frac{1024 \pi^{3}}{1215} \frac{\alpha^{2} n_{0}^{4}}{\hbar c^{2}}\left(\frac{T}{\mu}\right)^{4} ; \quad T \ll \mu .
$$

Its sublinear dependence on temperature reflects new regime of "cold" degenerate phonons. In the opposite limit $T \gg \mu$ we find linear law $\kappa \sim\left(\alpha^{2} n_{0}^{4} / \hbar c^{2}\right)(T / \mu)$ in agreement with Ref. [2, which is a result of the soliton interacting with "hot" classical phonons. The typical lifetime [15] of DS may be estimated from Eq. (1) as

$$
\tau=\frac{\left|M^{*}\right|}{\kappa}=\frac{1215}{256 \pi^{3}} \frac{\hbar^{2} c}{\alpha^{2} n_{0}^{3}}\left(\frac{\mu}{T}\right)^{4} .
$$

Taking ${ }^{87} \mathrm{Rb}$ atoms confined with transverse frequency $\omega_{\perp}=1 \mathrm{kHz}$ we find $g=10^{-39} \mathrm{~J} \mathrm{~m}$, and $-\alpha=$ $12 \log (4 / 3) g^{2} / \hbar \omega_{\perp} \simeq 3.5 \cdot 10^{-47} \mathrm{~J} \mathrm{~m}^{2}$, which for density $n_{0}=10^{8} \mathrm{~m}^{-1}$ and sound velocity $c \simeq 1 \mathrm{~mm} / \mathrm{s}$ yields $\tau \simeq 100 \mathrm{~ms} \times(\mu / T)^{4}$. Experimentally $\mu / T \sim 1$ so our results are in a reasonable agreement with the observed [5] soliton life-time of $\tau \sim 200 \mathrm{~ms}$.

We first discuss the nature of the soliton-phonon interactions. To this end we use the integrable case without the cubic in density $n^{3}$ term in the Lagrangian, and verify the absence of phonon emission by DS. We then reintroduce $\alpha$ and derive the dissipative force Eqs. (1), (3). The weakly interacting Bose gas may be described by the Lagrangian

$$
L=\int d x\left[\phi \partial_{t} n-\frac{\left(\partial_{x} \sqrt{n}\right)^{2}}{2 m}-\frac{n\left(\partial_{x} \phi\right)^{2}}{2 m}-\frac{g}{2}\left(n-n_{0}\right)^{2}\right],
$$

where $n(x, t)$, and $\phi(x, t)$ are density and phase fields. The gradient of the latter is related to the superfluid velocity $u(x, t)=\partial_{x} \phi(x, t) / m$, where $m$ is the mass of the bosons. In Eq. (5) we have subtracted the contribution of uniform density profile $n_{0}$. Variation of the Lagrangian (5) with respect to the fields gives the equations of motion

$$
\begin{aligned}
\partial_{t} n & =-\partial_{x} \frac{n}{m} \partial_{x} \phi \\
-\partial_{t} \phi & =-\frac{\partial_{x}^{2} \sqrt{n}}{2 m \sqrt{n}}+\frac{\left(\partial_{x} \phi\right)^{2}}{2 m}+g\left(n-n_{0}\right) .
\end{aligned}
$$

This is the hydrodynamic form of GP equation [16], Eq. (6) is the continuity equation, while Eq. (7) is the Euler equation. In the long wavelength limit, they reduce to the wave equation describing low energy sound waves (phonons) propagating with the sound velocity c. The latter depends on the background density $n_{0}$ and can be obtained from the thermodynamic relation $m c^{2} / n_{0}=\partial \mu / \partial n_{0}=g$. The corresponding Lagrangian written in terms of small variations of the density $\rho(x, t)=n(x, t)-n_{0}$ becomes

$$
L_{\mathrm{ph}}=\int d x\left[\phi \partial_{t} \rho-\frac{n_{0}+\rho}{2 m}\left(\partial_{x} \phi\right)^{2}-\frac{m c^{2}}{2 n_{0}} \rho^{2}\right] .
$$

The nonlinear phonon interactions term $\rho\left(\partial_{x} \phi\right)^{2}$ is retained since it is going to play an important role below.

In addition to the phonon modes, governed by Eq. (8), equations of motion (6), 77) support a one parameter family of soliton solutions [16] with the localized profiles 
of density $n_{\mathrm{s}}(x-V t ; V)$ and current $u_{\mathrm{s}}(x-V t ; V)$ given by

$$
\begin{aligned}
& n_{\mathrm{s}}(z ; V)=n_{0}\left(1-\frac{\sin ^{2}(\Phi / 2)}{\cosh ^{2}(z m c \sin (\Phi / 2))}\right) ; \\
& u_{\mathrm{s}}(z ; V)=V\left(1-\frac{n_{0}}{n_{\mathrm{s}}(z ; V)}\right) .
\end{aligned}
$$

The velocity $V$ is related to the the phase drop across the soliton $\Phi=\phi(-\infty)-\phi(+\infty)$ as $V / c=\cos (\Phi / 2)$. One also finds for the momentum and energy of the soliton

$$
P(V)=n_{0}(\Phi-\sin \Phi) ; \quad E(V)=E_{0} \sin ^{3}(\Phi / 2),
$$

where $E_{0}\left(n_{0}\right)=4 c n_{0} / 3$ can be regarded as the soliton chemical potential. Expressions (11) lead to the equation of motion for a soliton moving with a constant velocity $V=\partial_{V} E / \partial_{V} P=\partial E / \partial P$. In what follows we shall focus on dark, $\Phi \approx \pi$, small velocity solitons $V \ll c$, which have $P(V) \approx \pi n_{0}+M^{*} V$ and $E(V) \approx E_{0}+M^{*} V^{2} / 2[15$.

We now substitute the soliton solution, $n_{\mathrm{s}}(x-X ; V)$, and $u_{\mathrm{s}}(x-X ; V)$, Eqs. (9), 10, into Eq. (5). Here $X(t)$ is an instantaneous soliton coordinate related to the velocity $V$ by the equation of motion $\dot{X}=V$. As a result one finds an effective DS Lagrangian

$$
L_{s}=P(\dot{X}) \dot{X}-E(\dot{X})=\pi n_{0} \dot{X}+\frac{M^{*} \dot{X}^{2}}{2}-E_{0} .
$$

It provides Feynman path integral description of the soliton as a quantum particle with the coordinate $X(t)$ moving in constant background with density $n(x, t)=n_{0}$ and $u(x, t)=0$. Local fluctuations of density $n_{0} \rightarrow$ $n_{0}+\rho(X, t)$ and background velocity $u(X, t)$ interact with the soliton and modify its dynamics. In the longwavelength limit the effect of phonons can be studied by considering small changes to the uniform background. The change in the background density is accounted for by modifying the chemical potential of the soliton, the last term in Eq. (12), by expanding it as $E_{0}\left(n_{0}+\rho\right) \simeq E_{0}+$ $N_{0}\left(m c^{2} / n_{0}\right) \rho+\partial_{n_{0}}\left(N_{0} m c^{2} / n_{0}\right) \rho^{2} / 2$. Here $N_{0}=\partial E_{0} / \partial \mu$ is the number of particles expelled from the dark soliton. To obtain the coupling to the velocity field, we note that in the laboratory frame, where the liquid moves with the uniform velocity $u$, the fields Eqs. (9), 10 transform as $n_{\mathrm{s}}(z, V) \rightarrow n_{\mathrm{s}}(z, V-u), u_{\mathrm{s}}(z, V) \rightarrow u+u_{\mathrm{s}}(z, V-u)$. This transformation modifies momentum $P(V) \rightarrow P(V-u)$ and energy $E(V) \rightarrow E(V-u)+u P(V-u)$ of the soliton due to the uniform background flow $u$ [17]. Together with the density corrections it leads to the Lagrangian

$$
\begin{aligned}
L_{\mathrm{s}} & +L_{\mathrm{s}-\mathrm{ph}}=L_{\mathrm{s}}\left(\dot{X}-u, n_{0}+\rho\right)=-\pi\left(n_{0}+\rho\right)(\dot{X}-u) \\
& +\frac{M^{*} \dot{X}^{2}}{2}-M^{*} \dot{X} u+\frac{M^{*} u^{2}}{2}-2 c \rho-\frac{c \rho^{2}}{2 n_{0}},
\end{aligned}
$$

where $\rho=\rho(X, t)$ and $u=u(X, t)$.

Instead of tackling it directly, it is convenient to perform gauge transformation of the phonon density and velocity fields to get rid of terms linear in these variables. This is achieved by the following substitution $\rho(x, t) \rightarrow \rho(x, t)-N_{0} \delta(x-X(t))$ along with $u(x, t) \rightarrow$ $u(x, t)-(\pi / m) \delta(x-X(t))$. One should also redefine the soliton coordinate [18 $\dot{X}-\left(1+m N_{0} / M^{*}\right) u(X, t) \rightarrow \dot{X}$ to account for the phonon drag. Upon this change of variables in $L_{\mathrm{ph}}+L_{\mathrm{s}}+L_{\mathrm{s}-\mathrm{ph}}$ the soliton-phonon interaction Lagrangian acquires the form

$$
L_{\mathrm{s}-\mathrm{ph}}=-\frac{\Gamma_{\rho}}{2} \rho^{2}(X, t)-\frac{\Gamma_{u}}{2} u^{2}(X, t),
$$

where the soliton - two-phonon interaction vertices are given by

$$
\Gamma_{\rho}=\frac{\partial \mu}{\partial n_{0}} \frac{\partial N_{0}}{\partial n_{0}} ; \quad \Gamma_{u}=m N_{0}\left(1+\frac{m N_{0}}{M^{*}}\right) .
$$

Notice that to derive Eqs. (14) and 15) we crucially used the phonon non-linearity $m \rho u^{2} / 2$ in the Lagrangian (8). Since we have succeeded to transform the interaction Lagrangian to the form which does not contain terms linear in the phonon fields, we can disregard now the phonon non-linearity and treat them as the Luttinger liquid [19], described by the Gaussian part of Eq. 8) ( $\rho u^{2}$ term does not contribute to the leading temperature dependence).

We have arrived thus at the problem of the "quantum impurity" with the mass $M^{*}$, interacting with the Luttinger liquid through the two-phonon vertices (14). This problem was considered in Refs. [20] in close analogy to three dimensional dynamics of impurities in liquid ${ }^{4} \mathrm{He}$ [21]. The leading $\sim T^{4}$ contribution to the viscous force acting on the "impurity" corresponds to the process shown in Fig. 1 1a, where the "impurity" absorbs one thermal phonon, while emitting another long wavelength phonon to satisfy momentum and energy conservation. It results in equation of motion $M^{*} \dot{V}=F(V, 0)$, where the friction force exerted by the liquid on moving impurity is

$$
F(V, X)=-\frac{1}{4}\left(\Gamma_{\rho}-\Gamma_{u} \frac{c^{2}}{n_{0}^{2}}\right)^{2} \sum_{|q| \lesssim m c} e^{i q X} q \Pi(q, q V) .
$$

Here $\Pi(q, \omega)$ is the imaginary part of the Fourier transform of $\theta(t)\left\langle\left[\rho^{2}(x, t), \rho^{2}(0,0)\right]\right\rangle$ response function of the phonon gas. For small velocity, $V \ll c$, one finds

$$
\Pi(q, q V)=\frac{n_{0}^{2}}{8 m^{2} c^{3} T} \frac{q^{3}}{\sinh ^{2}(c q / 4 T)} V .
$$

The momentum sum in Eq. 16) is limited to $|q| \lesssim m c$. Indeed, phonons with the wavelengths shorter than DS size $(m c)^{-1}$ practically do not interact with the latter.

Using the relations $M^{*}=-2 m N_{0}=-4 n_{0} / c$ and $m c^{2} / n_{0}=g$ we find $\Gamma_{\rho}=c / n_{0}$ and $\Gamma_{u}=n_{0} / c$. In view of Eq. 16 $\dot{V}=0$, meaning that the soliton motion is unaffected by the interactions with the phonons. This is a consequence of the exact integrability of the Lagrangian in Eq. (5), which protects the soliton from the 
dissipation. This is also expected from the fact that the soliton configuration is an example of a reflectionless potential, playing an important role in the classical theory of integrable models [10], so it does not scatter phonons. Remarkably, in our approach this fact manifests itself through a subtle destructive interference of the phonons excited by the density and current vertices.

When a small cubic in density term $-\alpha\left(n-n_{0}\right)^{3} / 6$ is added to the Lagrangian (5), it breaks the exact integrability of the problem. Below we calculate the corrections to the Lagrangian (14) and demonstrate the lack of the exact cancelation of the prefactor in Eq. (16), leading to the dissipation and eventual evaporation of the soliton by emission of phonons. To find corrections linear in $\alpha$ to the soliton Lagrangian (12) it is sufficient to substitute the bare soliton configuration, Eqs. (9) and (10), into the cubic in density term,

$$
\delta L_{\mathrm{s}}=-\frac{\alpha}{6} \int d x\left[n_{s}(x ; V)-n_{0}\right]^{3}=\frac{8}{45} \frac{\alpha n_{0}^{3}}{m c}\left(1-\frac{V^{2}}{c^{2}}\right)^{\frac{5}{2}} .
$$

Expanding in $V / c \ll 1$, and comparing with Eq. $(12)$, one finds $\delta E_{0}=-8 \alpha n_{0}^{3} /(45 m c)$ and $\delta M^{*}=-8 \alpha n_{0}^{3} /\left(9 m c^{3}\right)$. To calculate corrections to the number of particles expelled from the soliton $N_{0}=\left(n_{0} / m c^{2}\right) \partial E_{0} / \partial n_{0}$ and its derivative which enters Eq. 15 it is important to take into account the renormalization of the sound velocity $m c^{2} / n_{0}=\partial \mu / \partial n_{0}=g+\alpha n_{0}$. We obtain the modified vertices

$$
\Gamma_{\rho}=\frac{c}{n_{0}}\left(1-\frac{2}{3} \frac{\alpha n_{0}^{2}}{m c^{2}}\right) ; \quad \Gamma_{u}=\frac{n_{0}}{c}\left(1+\frac{2}{9} \frac{\alpha n_{0}^{2}}{m c^{2}}\right),
$$

resulting in the non-zero soliton - two-phonon coupling in Eq. (16), $\Gamma_{\rho}-\Gamma_{u} c^{2} / n_{0}^{2}=-8 \alpha n_{0} /(9 m c)$.

To obtain Eqs. (1), (3) we notice that at small temperature $T \ll m c^{2}$ the momentum sum in Eq. (16) may be extended to infinity. Substituting then Eqs. (17) and (19) into Eq. 16), one finds $F(V, X)=-\kappa(T) V f\left(X / L_{T}\right)$, where

$$
\begin{array}{r}
f\left(\frac{y}{2 \pi}\right)=\frac{30}{\pi^{4}} \int d k \frac{k^{4} \cos (2 k y / \pi)}{\sinh ^{2} k}=\frac{15}{\sinh ^{5} y} \\
\times\left[\sinh y\left(3+2 \sinh ^{2} y\right)-y \cosh y\left(2+\cosh ^{2} y\right)\right] .
\end{array}
$$

The $T^{4}$ dependence of the friction force due to "cold" phonons with $q<T / c \ll m c[20$ ] is to be contrasted to the regime of larger temperatures $T \gg \mu \sim m c^{2}$ (still $\left.T \ll E_{0}\right)$ where all the phonons with $q \lesssim m c$ contribute to the dissipation, their number being simply proportional to $T$. One finds thus $\kappa \sim\left(\alpha n_{0}^{2} / m c^{2}\right)^{2} m T$ in agreement with the results of Ref. 2]. The numerical coefficient here depends on details of the momentum cutoff at $q \sim m c$ and is beyond our phenomenological long wavelength approach. To derive the equations of motion (2) for the gas of DS we describe interaction of each soliton with the phonons by the Lagrangian (14). Integrating out the Gaussian phonons and taking the semiclassical limit, we arrive at Eq. (2) with the force given by Eq. 20).

In conclusion, soliton scattering on background fluctuations, leads to its decay and a finite lifetime (unless the model is integrable). We have shown that in the quantum regime such a lifetime is significantly longer, than expected from classical considerations and solitons acquire long-range mutual interactions. Our approach may prove useful in other areas studying solitons propagation in a dynamic media, such as, e.g., non-linear optics.

The authors are grateful to M. Pustilnik for discussions which have initiated this project and to K. Bongs, M. Köhl and K. Matveev for fruitful discussions. D.M.G. acknowledges the hospitality of Prof. Dr. K. Sengstock's group in Hamburg and support by EPSRC Advanced Fellowship EP/D072514/1. A.K. was supported by NSF grant DMR-0804266 and EPSRC grant GR/T23725/01.

[1] W.P. Reinhardt and C.W. Clark, J. Phys. B 30, L785 (1997); Th. Busch and J.R. Anglin, Phys. Rev. Lett. 84, 2298 (1999); D.J. Frantzeskakis et al., Phys. Rev. A 66, 053608 (2002); V. V. Konotop and L. Pitaevskii, Phys. Rev. Lett. 93 (2004); N. Bilas and N. Pavloff, Phys. Rev. A 72, 033618 (2005);

[2] A. Muryshev et al., Phys. Rev. Lett. 89, 110401 (2002).

[3] B. Jackson, N.P. Proukakis, and C.F. Barenghi, Phys. Rev. A 75, 051601(R) (2007).

[4] S. Burger et al., Phys. Rev. Lett. 83, 5198 (1999); J. Denshclag et al., Science 287, 97 (2000); B. Anderson et al., Phys. Rev. Lett. 86, 2926 (2001).

[5] C. Becker et al., Nature Physics 4, 496 (2008).

[6] A.D. Jackson, G.M. Kavoulakis and C.J. Pethick, Phys. Rev. A 582417 (1998); A.E. Muryshev, H.B. van Linden van den Heuvell, and G.V. Shlyapnikov, Phys. Rev. A 60, R2665 (1999); D.L. Feder et al., Phys. Rev. A 62, 053606 (2000); J. Brand and W.P. Reinhardt, Phys. Rev. A 65, 043612 (2002); G. Theocharis et al., Phys. Rev. A 76, 045601 (2007).

[7] E. H. Lieb and W. Liniger, Phys. Rev. 130, 1605 (1963).

[8] P. Kulish, S. Manakov, and L. Faddeev, Theor. Math. Phys. 28, 615 (1976).

[9] E. H. Lieb, Phys. Rev. 130, 1616 (1963).

[10] L. D. Faddeev and L. A. Takhtajan, Hamiltonian Methods in the Theory of Solitons (Springer, Berlin, Heidelberg, 1987).

[11] J. Dziarmaga, Phys. Rev. A 70063616 (2004).

[12] A. Negretti, C. Henkel, and K. Mølmer, Phys. Rev. A 78023630 (2008).

[13] I. E. Mazets, T. Schumm, and J. Schmiedmayer, Phys. Rev. Lett. 100210403 (2008).

[14] According to fluctuation-dissipation theorem, the friction force is accompanied by stochastic force $\xi(t)$ with the correlator $\left\langle\xi(t) \xi\left(t^{\prime}\right)\right\rangle=2 \kappa T \delta\left(t-t^{\prime}\right)$. The complete semiclassical description of the soliton dynamics is given by the distribution function, satisfying the Fokker-Planck equation. Here we restrict ourselves with the dynamics of the maximum of this distribution only. 
[15] Eq. (4), based on Eqs. (1), (3) is valid in the experimentally most relevant regime $V \ll c$. A more general case, which includes the $\mathrm{KdV}$ regime $V \lesssim c$ is treated elsewhere 22.

[16] L. P. Pitaevskii and S. Stringari, Bose-Einstein Condensation (Clarendon Press, Oxford, 2003).

[17] This transformation shows that DS behaves as a particle with zero bare mass $M=0$, but finite effective mass $M^{*}$.

[18] The corresponding transformation of the arguments of $\rho(X, t)$ and $u(X, t)$ leads to higher order interactions, not contributing to the leading small temperature result.

[19] F. M. D. Haldane, Phys. Rev. Lett. 47, 1840 (1981).

[20] A. H. Castro Neto and M.P.A. Fisher, Phys. Rev. B 53, 9713 (1996); D. M. Gangardt and A. Kamenev, Phys. Rev. Lett. 102, 070402 (2009).

[21] L. D. Landau and I. M. Khalatnikov, Zh. Eksp. Teor. Fiz. 19, 637 (1949).

[22] D.M. Gangardt and A. Kamenev, in preparation. 